\title{
تصميم نظم معالجة بتقنيات مبسطة لمياه الصرف الصحي (دراسة حالة)
}

\author{
د. عبد المجيد ختريش 1 و أ. ناجي شكثم2 \\ 2،1 قسم الهندسة المدنية، كلية الهندسة، جامعة صبراتة، صبراتة، ليبيا. \\ ${ }^{1}$ khitreesh@yahoo.com
}

الملخص

تعد مياه الصرف الصحي من أكبر مصادر التلوث البيئي على المستوى العالمي من حيث الحجم، ومع الزيادة المستمرة في معدلات استهلاك المياه نتيجة زيادة عدد السكان والتوسع العمراني، ومع محدودية قدرات التخطيط والتمويل اللازمة لإقامة محطات متقدمة لمعالجة مياه الصرف الصحي للعديد من التجمعات السكانية في ليبيا دعت الحاجة لدراسة واستخدام طرق رخيصة وفعالة لمعالجة مياه الصرف الناتجة من هذه التجمعات قبل تصريفها.

تهدف الدارسة إلى تصميم منظومة لمعالجة مياه الصرف الصحي بتقنية مبسطة لمشروع إسكاني يقع جنوب مدينة صرمان لمعالجة تدفق يصل إلى 750٪/يومياً، وتتكون هذه المنظومة من نموذج معالجة يعمل بتقنية حوض إمهوف، ومن أهم مميزات هذا النظام محدودية التكلفة، وسرعة التنفيذ، والجمع بين فصل المواد الصلبة عن السوائل، وتثبيت الحمأة في وحدة واحدة. أما المياه المعالجة من حوض إمهوف يتم تصريفها خلال خنادق تحتوي على خطوط أنابيب ممتدة تحت الأرض بعمق 1.25 م وبقطر لا يقل عن 10 سم يستفاد منها في ري أشجار الغابات وتحسين البيئة المحيطة بالموقع.

لقد أسفرت الدراسة إلى أنه بتصميم حوض بسعة حوالي 33033، وبمساحة 3.5 هكتار لخنادق تصريف المياه المعالجة يمكن معالجة مياه الصرف الصحي لهذ التجمع السكاني وإلى حين ربطه مع كامل منظومة المعالجة بالمدينة في المستقبل.

الكلمات الرئيسية: معالجة مياه الصرف الصحي، تقنيات المعالجة، حوض إمهوف. 


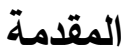

تهدف عملية معالجة مياه الصرف الصحي إلى إزالة الملوثات الفيزيائية، والكيميائية، والبيولوجية من مياه الصرف الصحي لحماية الصحة العامة والبيئة من التلوث، إضافة إلى الحصول على مياه نقية يمكن إعادة استخدامها في أغراض متعددة، إن المياه الملوثة اللامركزية يمكن أن تتتج عن بيت واحد، أو تجمع تجارى، أو سياحي، أو عن تجمع لعشرات المنازل المخدومة، أو غير المخدومة بشبكة صرف صحي كما في القرى، وفى العديد من البلدان النامية هناك نقص في شبكات الصرف الصحي وبالتالي يكون صرف مياه الصرف الصحي عشوائيا على الأغلب (التركماني 2009) ولكي يتم تفادى النتائج السلبية لهذه المياه الملوثة على البيئة والصحة العامة فمن الواجب معالجة هذه المياه الملوثة قبل تصريفها إلى الطبيعة.

إن السعي نحو الربط الثامل للقرى والتجمعات السكانية الصغيرة المتباعدة بشبكات صرف صحي موحدة يعنى إنفاق موارد مالية كبيرة جداً على هذه الثبكات بغض النظر عن كلفة المعالجة. وإن تطبيق المعالجة بمكان ومصدر التلوث أو الاعتماد على وسائل بديلة عن نظام الصرف الصحي التقليدي أعطى حلولاً عملية لتفادي الإنفاق الهائل على مشروعات الصرف الصحي ومحطات المعالجة حيث بلغت نسب التوفير المالية بحدود 40-70 \% (الأمم المتحدة 2000).

إن الحلول المستدامة للتجمعات الصغيرة اللامركزية يعنى توفير الكلفة وتجنب تلوث المياه السطحية وتلوث مصادر مياه الثرب، ويعنى توفير الجهد، وتقليل المخاطر البيئية المحتملة، وأن قرب مكان

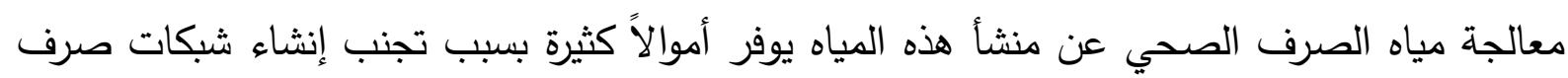
صحي ذات أطوال كبيرة. إن محطات المعالجة عادة ما يتم تقييمها على أساس الحفاظ على الصحة العامة وحماية البيئة أكثر من تقييمها على اعتبارات اقتصادية وهذا لا يعفي من البحث عن أسلوب المعالجة الأكثر اقتصادية الذي يحقق المعايير البيئية المطلوبة، وإن الحديث عن معالجة مياه الصرف الصحي للمناطق اللامركزية يعني بساطة طرق المعالجة، وسهولة التثغيل والصيانة (الفلاح 2005). 
مشكلة الدراسة

$$
\text { • ماهي طرق معالجة الصرف الصحي؟ }
$$

كيف يتم اختيار الطرق المثلة لمعالجة المياه العادمة من الوحدات السكنية؟

$$
\text { ماهي خصائص حوض إمهوف لمعالجة الصرف الصحي؟ }
$$$$
\text { ما هي مميزات وعيوب خزان إمهوف لمعالجة الصرف الصحي؟ }
$$

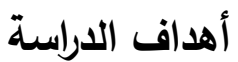

اختيار وتصميم نظام معالجة مبسط لمياه الصرف الصحي الناتجة من التجمع السكاني. •أبراز مشاكل عدم استكمال محطة المعالجة المركزية لمدينة صرمان. استعراض بعض من الطرق العلمية لمعالجة مياه الصرف الصحي.

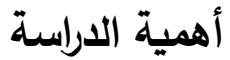

تصريف مياه الصرف الصحي الناتجة من التجمع السكاني بمنطقة الدراسة بطريقة اقتصادية وآمنة. استخدام تقنيات مبسطة واقتصادية بالموقع لمعالجة الصرف الصحي.

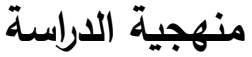

تعتبر هذه الدراسة حالة دراسية حيث اعتمدت على الدراسة المسحية للموقع من أعداد السكان المتوقعة للوحدات السكنية وكذلك الوحدات المكونة لمحطة المعالجة، كما اهتمت الدراسة بدراسات مشابهة في العالم ومقارنتها بالدراسة الحالية. ومن ضمن الأدوات المستخدمة في هذا البحث بعض البرامج الحاسوبية المساعدة في تصميم الوحدات المكونة للمشروع.

الادراسات السابقة

- تثير دراسة (Lee boon,other,2015) إلى إيجاد حلول للمشاكل التشغيلية التي تواجه تشغيل أحواض Imhoff 
الفلتر القديمة واستبدالها بطبقة جديدة، أما من الناحية الاقتصادية يعتبر مكلف ويمكن إيقاف عمل الخزان لفترة حتى تتم الصيانة. ولكن في السنوات الماضية تم استخدام بيروكسيد الهيدروجين المحفز catalyzed hydrogen peroxide) CHP) وهو مركب غير عضوي مؤكسد ذو فاعلية قوية في فصل المواد الصلبة عن السوائل حيث تثير النتائج إلى أنه بعد استعماله في حل مشاكل الانسداد أعطت نتائج جيدة بشكل ملحوظ في تقليل الانسداد بشكل كبير - استعرضت الورق (Anne Mikelonis,2008) تجربة دولة الهندوراس (2007) في تتفيذ وتثغيل عدد 18 حوض إمهوف موزعة على المناطق النائية حيث أدت الأحواض دورا مهما في المناطق التي تفتقر إلى مساحات شاسعه من الأراضي المسطحة بالإضافة إلى ذلك فهي إنثاءات ملموسه تمثل استثمارا مهما سابقا في البنية التحتية، وتظل خزانات إمهوف خيارا عمليا للعلاج في بعض المجتمعات النامية لعدة أسباب وأهمها منخفض التكلفة، أقل استهلاكا للطاقة مقارنة بالأساليب الأخرى مثل معالجة الحمأة المنشطة. - أجرت دراسة (Yashashri Patil,2018) على أنواع الغازات الناتجة من حوض إمهوف واوضحت أنه بالإمكان الحصول على عدة أنواع من الغازات وأههها (كبريتيد الهيدروجين, ثاني

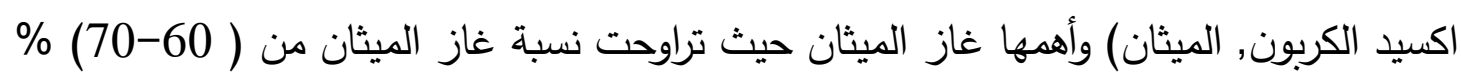
ويمكن استخدام هذا الغاز للأغراض التالية: تسخين المياه للتدفئة، إنتاج الطاقة الكهبائية.

تبين الدراسة الأولى أهمية الخبرة في التعامل مع تثغيل حوض إمهوف والبحث عن الحلول للمشاكل المتوقع حدوثها أثناء تشغيل الخزانات وخاصة مشاكل تعطل فلتر التصفية، والدراسة الثانية توضح تجربة دولة الهندوراس، وكذلك تبين خصائص ومميزات خزانات إمهوف، أما الدراسة الثالثة فلها أهمية في عرض الغازات الناتجة من خزانات إمهوف وكيفية الاستفادة من غاز الميثان في التدفئة وتوليد الطاقة.

\section{أنظمة وإختيار أسلوب معالجة مياه الصرف الصحي}

إن أسلوب المعالجة المختار ونوع المنشآت وطريقة الاستثمار يؤثر كثير على سلامة البيئة المحيطة بالمحطة خصوصا عندما يتم اختيار نظام أو أسلوب غير مناسب للمكان أو البيئة السكانية والاجتماعية 
Metcalf ) ويمكن تصنيف أنظمة معالجة الصرف الصحي من حيث الخصائص التي تميز كل نوع إلى :(\& Eddy 2003

أ- - نظم المعالجة الميكانيكية: وهي الأنظمة التي تستخدم في المدن والتجمعات السكانية الكبيرة وهى تمتاز باستهلاك عالٍ للطاقة بالإضافة إلى احتياجها للمعدات الميكانيكية والأحواض الخرسانية في عملها وعادة هذه الأنظمة تتشأ على مساحات أرض صغيرة _ مثل الحمأة المنشطة وكافة تطبيقاتها مثل قنوات الأكسدة، المرشحات الحجرية. ب- - نظم المعالجة الطبيعية: هذا النوع من المعالجة الطبيعية يعتمد على الظروف الطبيعية (كالثمس والرياح) للحصول على المعالجة المطلوبة لمياه الصرف الصحي وذلك بدون الاحتياج إلى تدخل خارجي كإعطاء طاقة للمساعدة في إتمام المعالجة أو بدون الاستعانة باستهلاك ضخم من الطاقة أو المواد الكيمياوية، وهذا النوع من الأنظمة يحتاج إلى مساحات كبيرة من الأراضي للإنشاء مما يجعله غير مناسب للاستخدام في المناطق ذات الكثافة السكانية العالية أو عندما يكون سعر الأرض غالياً، كما يمتاز هذا النوع بأنه ذو تكلفة منخفضة نسبيا (عند توفر الأرض) وسهولة التثغيل والصيانة للمنثأ محدودة. وعادة فإن هذا النوع من المعالجة الطبيعية يكون أكثر كفاءة في إزالة الجراثيم الممرضة عن أنظمة المعالجة الميكانيكية. مثل: بحيرات الأكسدة، أحواض إمهوف، برك النباتات العالقة، الأراضي الرطبة، المعالجة عن طريق التصريف من خلال الأرض.

\section{أنظمة المعالجة بالموقع مقابل أنظمة المعالجة خارج الموقع}

يتكون نظام المياه الملوثة من ثلاثة مكونـات رئيسية وهي: النقل، والمعالجة، والمخلفات الصلبة، وإن العنصـر الأقل أهميـة هو موضـوع نقل مياه الصـرف الصـحي إذا مـا قورن مـع معالجـة ميـاه الصـرف الصحي وطريقة التخلص من المياه المعالجة والمخلفات الصلبة بثكل آمن، أن الأنابيب لا تعالج المياه الملوثة ومع هذا فإن الكلفة العظمى لمشروع الصرف الصحي المركزي (شبكة مع محطة معالجة) تكون Gijzen, من نصيب شبكة الصرف بحيث تبلغ كلفتها أكثر من 60\% من الكلفة الكلية للمشروع 2003)، إن قرب مكان معالجة مياه لصرف الصحي عن منشأ هذه المياه يوفر أموالاً طائلة بسبب تجنب إنثاء شبكات صرف صحي ذات أطوال كبيرة. وإن الحديث عن معالجة مياه الصرف للمناطق 
اللامركزية يعني بساطة طرق المعالجة، وسهولة التشغيل، والصيانة؛ لتجنب الحاجة إلى خبرات ماهرة في التشغيل والصيانة التي عادة لا تكون متوفرة في البلدان النامية. إن المعالجة المطبقة في مكان مصدر التلوث أعطى حلولاً عملية لتفادي الإنفاق الهائل على مشروعات الصرف الصحي ومحطات المعالجة. ومن أهم ميزات أنظمة المعالجة بالمكان أن مياه الصرف المنزلي تعالج قرب مصدرها مع إمكانية استخدام التقنيات الحديثة عبر المحطات الصغيرة. وأن التخلص من المياه المعالجة يكون عبر شبكة أنابيب أو فلتر حصوي تحت الأرض مع أمكانية تصريف المياه المعالجة إلى المسطحات المائية أو الأنهار أو استخدامها بالري. كما أن المعالجة المنفردة لمياه الصرف الصحي تعطي عدم تركيز المغذيات الموجودة بالمياه المعالجة في مكان واحد (المصرف النهائي) وهذا له دور كبير في تخفيض تأثير المغذيات على نوعية المياه المستقبلة وعادة ما تستخدم المياه في أنظمة إدارة مياه الصرف في الموقع بعد المعالجة لتتظيف دورات المياه وأغراض الري وغسيل الشوارع.... الخ. إن السؤال المطروح هو مدى جدوى تجميع المياه الملوثة في التجمعات القروية عبر شبكة صرف صحي المركزية مما يتسبب بتجمع كمية كبيرة من مياه الصرف الصحي وبالتالي تصبح الحاجة إلى محطات معالجة ذات كلف مادية كبيرة. وإن الاختلافين الرئيسين بين أنظمة المعالجة بالمكان والنظام التقليدي هما نظام (شبكة) نقل مياه الصرف وتجميعها ونوع نظام التخلص النهائي من المياه المعالجة والمخلفات الصلبة. إن من أهم معايير الاختيار للمعالجة بالموقع أو المعالجة خارج الموقع هي الكثافة السكانية (عدد السكان في كل هكتار أرض) وحجم مياه الصرف الصحي المنتجة (بالمتر المكعب /هكتار/يوم) ووجود آبار مياه ضحلة معرضة للتلوث بالصرف الصحي، نفاذية التربة وكذلك وحدة نظام خدمة الصرف الصحي أيضاً تعتبر عوامل رئيسية وتقوم الاعتبارات الاجتماعية بذور مهم في اختيار نظام الصرف الصحي، خاصة في نظم المعالجة بالموقع. فالفروق الثقافية والمحلية (المفاهيم الثقافية عن المخلفات البشرية) يمكن أن ينشأ عنها قبول وفعالية النظام في مكان محدد وعدم قبوله في مكان آخر • وعلى الأغلب فإن أخذ القرار بالأسلوب الأنسب للمعالجة لأي تجمع من التجمعات الصغيرة أو اللامركزية يتم بناءً على خيارات متاحة وظروف تتعلق بالكلفة والطاقة اللازمة والأرض المطلوبة. بالإضافة إلى متطلبات عامة لمحطة معالجة مياه الصرف الصحي للتجمعات السكانية الصغيرة التي تتضمن: 


\section{• • سهولة صيانة المعدات مع الحد الأدنى من الطاقة اللازمة.}

• زمن مكوث طويل نسبيا وبالتالي استيعاب عملية تذبذب التدفقات طوال اليوم التي تميز التجمعات السكانية الصغيرة وبالتالي تجنب حدوث الصدمات الهيدروليكية. يجب أن يكون تشغيل المحطة فعالاً لأجل مجال واسع من الحمولات العضوية والهيدروليكية القدرة على مواجهة الحالات الطارئة (انقطاع التيار الكهربائي مثلا). • • حقيق مواصفات خاصة للمياه المعالجة وبأقل كلفة مدكنة. نوعية و كمية المياه الملوثة. • البيئة المحيطة ومكان تصريف المياه المعالجة. تقنيات إدارة المياه الملوثة (نظام النقل و التجميع و المعالجة و التصريف النهائي). التشغيل والصيانة والبنى التحتية المتوفرة.

وفي الدول المتقدمة فإن تقنيات إدارة مياه الصرف الصحي بالمكان قد تحددت سماتها وأصبحت تتمتع بدراسات وأبحاث هندسية كافية ومتطورة، وبات من الممنوع إقامة الأنظمة المركزية لنقل ومعالجة مياه الصرف الصحي بحالة إمكانية تطبيق أنظمة المعالجة بالمكان إلا في الحالات القاهرة، وعبر السنوات الطويلة فقد ترسخت القناعة بأنه أذا توفر حسن التخطيط والتصميم والتتفيذ والإدارة والتثغيل لأنظمة معالجة مياه الصرف الصحي في الموقع فإن الإدارة طويلة الأمد لمياه الصرف الصحي ستكون الحل البديل والمنافس الاقتصادي للأنظمة المركزية (Gijzen, 2003).

\section{وصف موجز لمنطقة الدراسة}

يعتبر مشروع ألف وحدة سكنية بجنوب مدينة صرمان من ضمن المشاريع التتموية التي شُرع في تتفيذها بمختلف المناطق الليبية سنة 2008 وهو يستهدف تصميم وتتفيذ عدد ألف وحدة سكنية بمساحات متعددة متمثلة في خمسة نماذج، كما يشمل المشروع مجموعة من المباني والمرافق الخدمية وجميع مكونات البنية التحتية بمساحة أجمالية 91 هكتار، وقد تم تصميم تصريف مياه الصرف الصحي في نقطة منخفضة عن منسوب المشروع ليتم ضخها إلى محطة معالجة مياه الصرف الصحي وهي تحت 
التنفيذ في تلك الفترة، ويقع المشروع جنوب شرق المدينة بمنطقة شبه صحراوية على بعد حوالى 4 كم من وسط مدينة. الثكل (1) يوضح موقع المشروع (محطة المعالجة والحي السكني).
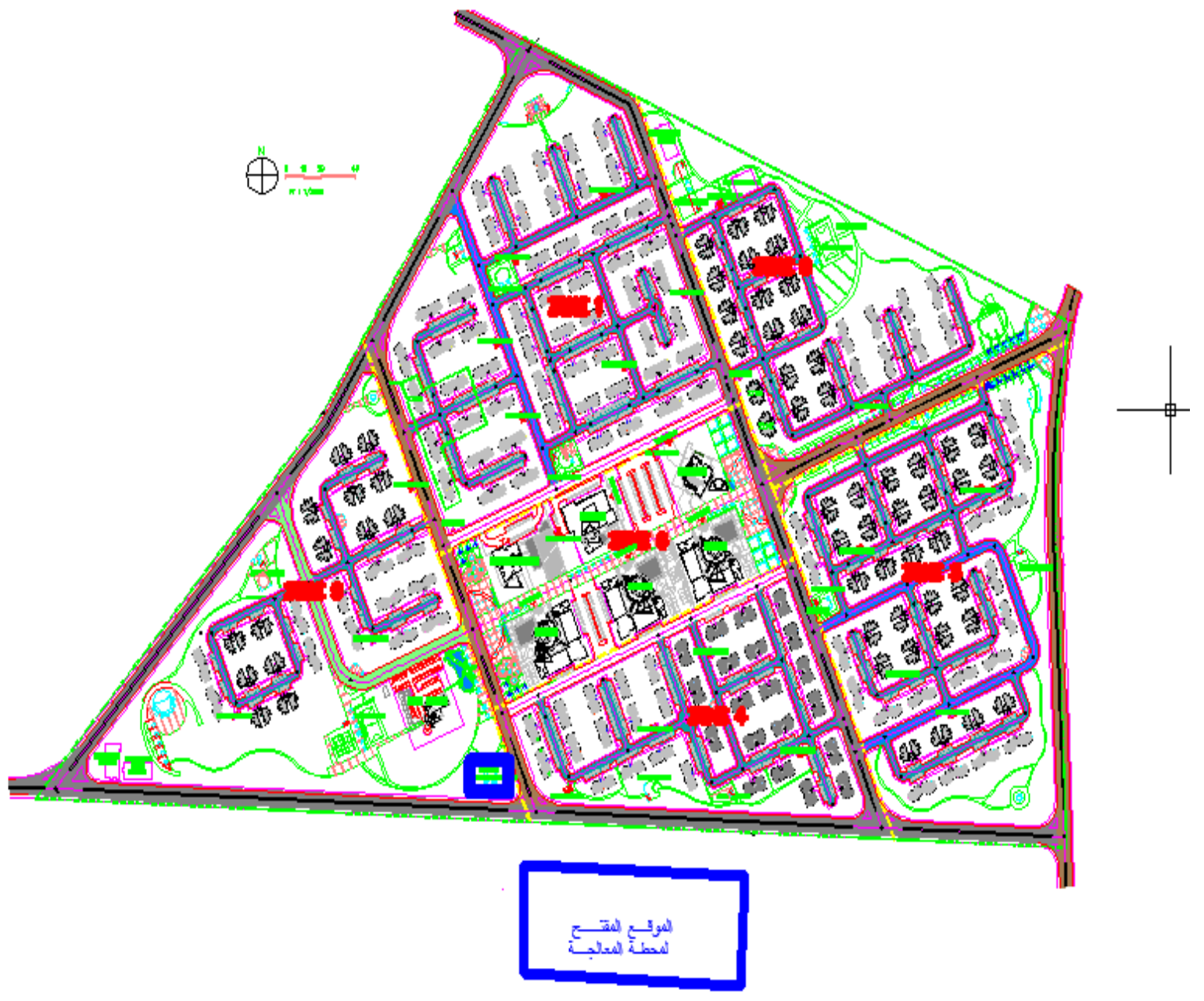

شكل (1): موقع المشروع يبين محطة المعالجة والحي السكني.

تم البدء في تتفيذ المحطة سنة 2000م، وتم الانتهاء منها سنة 2007 وهي تعمل بطريقة الحمأة المنشطة (تقنية قنوات الأكسدة) (الثركة العامة للمياه والصرف الصحي 2014). ومنذ ذلك الحين لم يتم تجربة واستلام المحطة بسبب عدم استكمال مشروع تصريف مياه الصرف الصحي الذي يشمل ربط الوحدات السكنية بالشبكة كما هو مبين بالشكل (2) الذي يمثل المخطط العام للمشروع السكني، وقد 
صمدت المحطة بطاقة استيعابية تقدر (10000 م3/يوم) لاستيعاب مياه الصرف لكامل سكان المدينة حتى عام 2020.

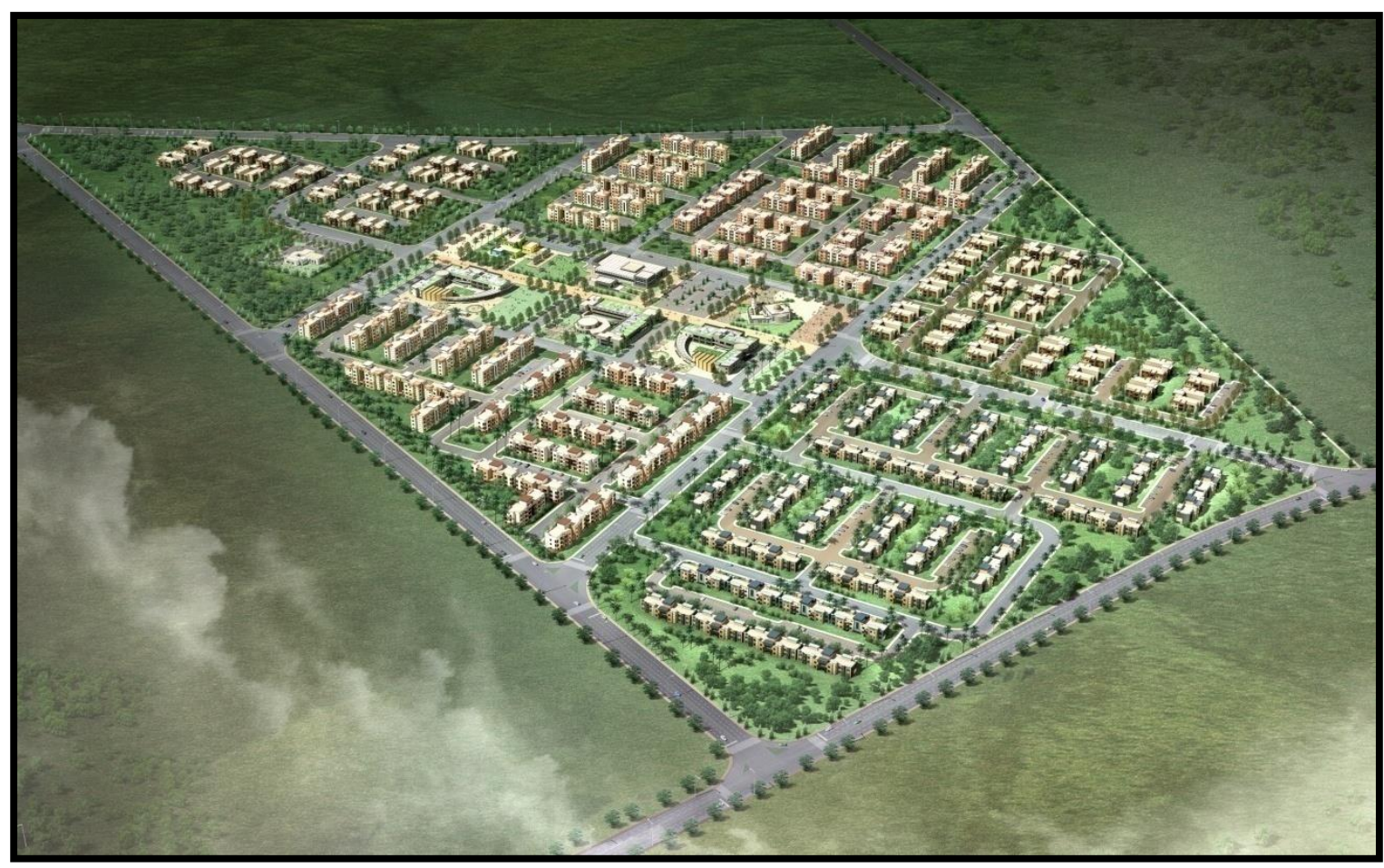

شكل (2): المخطط العام لمشروع الحي السكنى ألف وحدة سكنية جنوب صرمان.

\section{اختيار التصميم والتقنية المناسبة لمنطقة الدراسة}

تم في هذه الدراسة اختيار تقنية حوض إمهوف Imhoff Tank لمعالجة مياه الصرف الناتج من التجمع السكاني (ألف وحدة سكنية)، وهي تقنية للمعالجة الابتدائية لمياه الصرف الصحي الخام وهي مصممة لفصل المواد الصلبة عن السوائل وهضم الحمأة (Metcalf \& Eddy 2003)، حيث يتم الجمع بين فصل المواد الصلبة عن السوائل وتثبيت الحمأة في وحدة واحدة. وهو يعمل على نفس آلية خزان التحليل ولكن يستعمل لتدفق أعلى من خزنات التحليل، وينصح باستخدامه لتدفقات مياه الصرف المنزلية أو المختلطة لتعدادات سكانية تبلغ ما بين 20 إلى 50 ألف نسمة، وهي قادرة على مُعالجة أحمال عضوية عالية، وتكون مُقاومة أيضًا للأحمال العضوية المفاجئة. ومتطلبات المساحة لأحواض إمهوف صغيرة، وتكون تكاليف التنفيذ أقل مقارنة بتكاليف معالجة مياه الصرف الصحي الأخرى. وكذلك تكاليف التشغيل 
مُنخفضة حيث لا تحتاج إلى معدات ميكانيكية معقدة وعمالة فنيه ماهرة. ويمكن استخدام المياه المعالجة من حوض إمهوف في ري أثجار الغابات وتحسين البيئة العامة.

إن حوض إمهوف هو حوض ترسيب قوي وفعَّال حيث يخفض المواد العالقة بنسبة ما بين 50 إلى 60\% ويخفض الأكسين الحيوي المستهلك BOD ما بين 25 إلى 45\%، ويؤدي إلى تثبيت الحمأة بشكل جيد، وهذا يعتمد على التصميم والظروف المحيطة.

\section{اعتبارات التصميم لاستخدام تقنية حوض إمهوف}

يتكون حوض إمهوف كما هو مبين بالثكل (3) من جزء ترسيب على شكل حرف (V) قاعه مائل بدرجة كبيرة فوق حجرة لتجميع وهضم الحمأة مع جزء لتنفيس الغاز • بحيث يتم ترسيب المواد الصلبة الأثقل على شكل حمأة، أما المواد الخفيفة مثل الثحوم الدهون فتبقى طافية مكونة طبقة من الخبث على سطح المياه في حين تحمل المواد المتبقية في الخزان إلى عمليات التحلل اللاهوائي بواسطة النشاط البكتيري مما يسبسب انخفاض كبير في حجم الحمأة المتبقية، وهذا بدوره يسمح للخزان بفترات تثغيل قد تمتد من سنة إلى أربع سنوات قبل أن يحتاج إلى التنظيف.

الجزء الخاص بالترسيب له شكل دائري أو مستطيل ذو جدران على شكل حرف V، وفتحة في الجزء الهُُفلي؛ مما يسهح للمواد الصلبة بالترسب والاستقرار في الجزء الخاص بالهضم ويَمَع الغاز من الارتفاع لأعلى وعمل اضطراب لعملية الترسيب. والغاز اللُّنتج في حجرة الهضم يرتفع إلى فتحات الغاز على حافة الدُفاعل، حيث يقوم بنقل جُزئات الحمأة إلى سطح الماء مُكونًا طبقة من الخبث (الزبد). وتتراكم الرواسب في حُجرة هضم الحمأة، ويتم ضغطها وتثبيتها جُزئيًا من خلال الهضم اللاهوائي.

ويتم بناء حوض إمهوف عادة تحت الأرض بالخرسانة المسلحة، ويُكن أيضًا أن يُنيى فوق سطح الأرض، مما يجعل إزالة الحمأة أسهل بسبب الجاذبية، ولكنه في هذه الحالة يحتاج إلى ضَخ التدفقات السائلة الداخلة إليه من أعلى، زمن البقاء الهيدروليكي (HRT) في العادة لا يكون أكثر من 2 إلى 4 الكي ساعات للحفاظ على مياه التدفقات السائلة الخارجة بخصائص هوائية مناسبة للمُعالجة التالية أو 
للتصريف (Imhof, B. and Muhlemann, 2005). وتوضع أنابيب على شكل حرف T أو حواجز على مدخل ومخرج الحوض لخفض سرعة الماء الداخل ولمنع الخبث من مغادرة نظام المُعالجة. معدل التحميل السطحي للغرفة العليا يكون 20-30 م|/2/20وم، أما عرض الحوض العلوي الخاص بالترسيب فيكون بين (70 - 75 \%) من العرض الكلى للحوض، في حين العمق الكلى للحوض لا يقل عن 5 متر، ويجب أن تكون جُدران حُجرة هضم الحمأة مائلة بدرجة 45ه أو أكثر، وهذا يَسمح للحمأة أن تنزلق إلى المركز حيث يُمكن إزالتها، وأبعاد حُجرة الهضم اللاهوائي تعتمد في الأساس على معدل إنتاج الحمأة بالنسبة للتعداد السُكاني، وعلى درجة التثبيت المُستهدفة للحمأة (المُرتبطة بالمُعدل الدوري لإزالة الحمأة) ودرجة الحرارة. وتُصمم السعة التخزينية لحُجرة هضم الحمأة لمدة تتراوح من 4 أشهر إلى 12 شهرًا، وذلك للسماح بالهضم اللاهوائي الكافي. وفي الكُناخات الباردة تحتاج الحمأة لزمن بقاء أطول، وبالتالي هناك حاجة إلى حجم أكبر للحَوْض. ولإزالة الحمأة يجب أن يتم تركيب أنبوب ومَضخة لسحب الحمأة من الحَوْض، أو السماح لشاحنات الثفط والمَضخات المُتتقلة بأداء هذه المهمة. ولمنع المواد كبيرة الحجم من التأثير على نظام اليُعالجة فإنه يُنصح باستخدام مصفاة قضبانية أو غُرفة حجز الحصى كما هو الحال في المعالجة الأولية قبل حَوْض إمهوف.
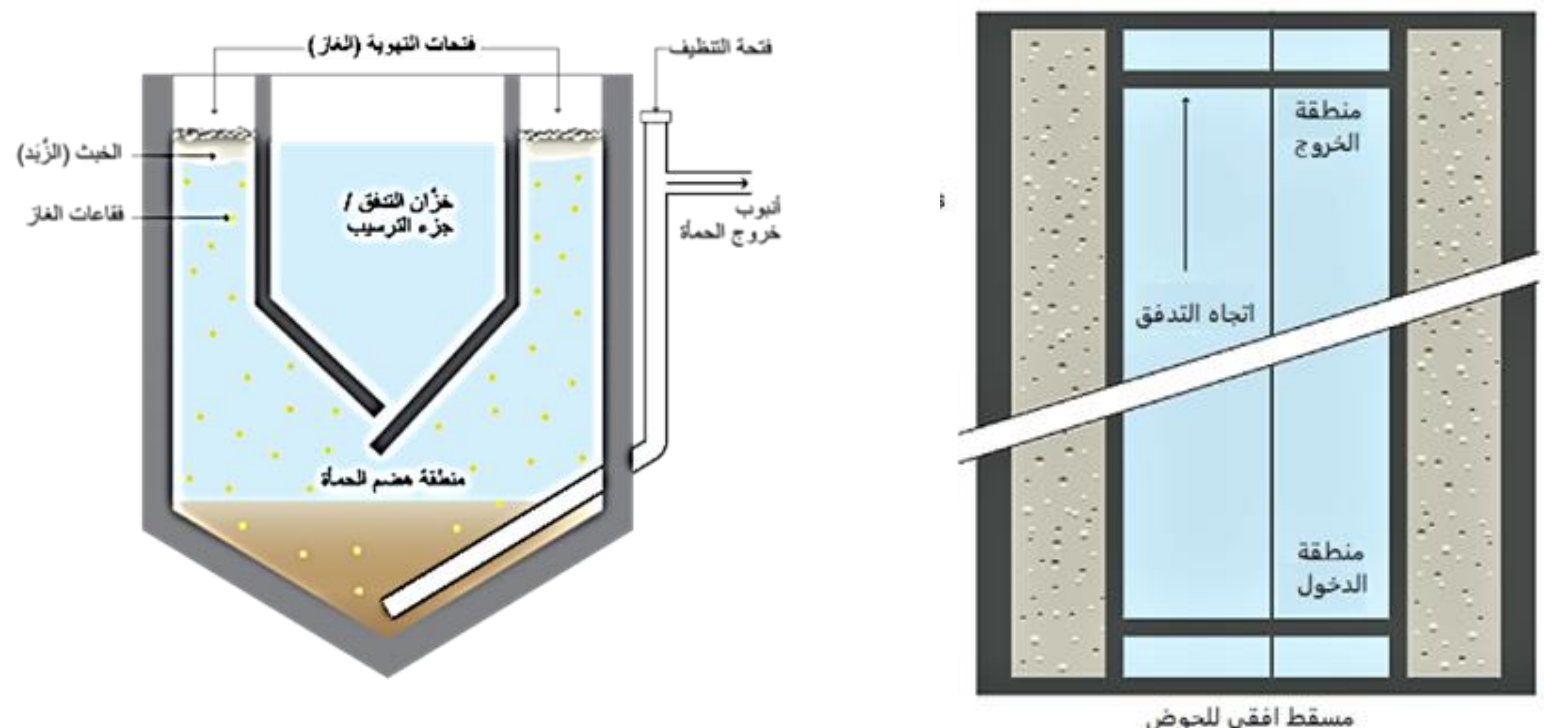

مسقط افقى للحوض

شكل (3): مقطع ومسقط أفقي لحوض إمهوف (Metcalf \& Eddy, 2003). 


\section{الجوانب الصحية والتأثيرات البيئية لتقنية استخدام حوض إمهوف بمنطقة الدراسة}

يتكون نظام المعالجة المقترح من حوض إمهوف متبوع بمنطقة من خنادق التصريف ممتدة تحت سطح الأرض بما لا يزيد عن 1.25 متر وبقطر لا يقل عن 10 سم محاطة بطبقة من الركام تعلوها طبقة سطحية عازلة يتم ردمها بالتربة العادية الموجودة بالموقع بمساحة تبلغ حوالى 14 هكتار لكامل التعداد السكاني 6000 شخص (Metcalf \& Eddy 2003)، وهذا الحل يساعد على التخلص من جميع مخرجات الحوض بشكل آمن التي منها إزالة لمسببات الأمراض، أما بعض المشاكل الأخرى مثل مشاكل ظهور رغوة نتيجة لتغير في درجة الحرارة أو الارتفاع المفاجئ في الأحمال العضوية، أو الغازات التي تظهر بكميات منخفضة التي يمكن أن تولد روائح غير مرغوبة بها، فيمكن حل هذه المشاكل بواسطة فريق مدرب من خلال الاهتمام بالتشغيل والصيانة الجيدة، بحيث تبقى مسارات التدفق مفتوحة وتتظف أسبوعيا (Imhof, B. and Muhlemann 2005)، مع إزالة الخبث في جزء الترسيب وفتحات التهوية، وإزالة الحمأة المثبتة في قاع الحوض حسب المدة المقترحة بالتصميم المطبق. مع التأكد طوال الوقت من وجود 50 سم على الأقل تقصل بين الحمأة المترسبة وفتحة جزء الترسيب لضمان حسن أداء الحوض.

\section{معايير التصميم والكميات التقريبية لإنشاء خزان إمهوف للتجمع السكاني}

الجدول رقم (1) يبين معايير التصميم الأولية لحوض إمهوف، بينما الجدول رقم (2) يبين النتائج التي تم التوصل إليها لأبعاد حوض إمهوف المقترح للتجمع السكاني ألف وحدة سكنية بكامل مرافقه. أما الجدول رقم (3) فيبين الكميات التقريبية لتنفيذ التصميم المقترح بدون حساب ثمن الأرض الذي يشمل تكاليف إنثاء حوض إمهوف بسعة 330 م3، وتكاليف توريد ومد جزء من شبكة أنابيب تحت الأرض لتصريف مياه الصرف الناتجة من المعالجة الأولية بحوض إمهوف وبعض التكاليف الإضافية لتشغيل

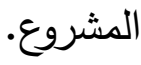


جدول (1): معايير التصميم الأولية لحوض إمهوف

\begin{tabular}{|c|c|}
\hline \multicolumn{2}{|c|}{ معايير التصميم الأولية } \\
\hline 6000 شخص & عدد السكان \\
\hline 150 لتر للفرد & متوسط الاستهلاك اليومي للمياه \\
\hline 250 ملجم/ لتر & الأكسجين الحيوي المستهلك BOD \\
\hline 333 ملجم/تتر & مجموع المواد الصلبة العالقة TSS \\
\hline
\end{tabular}

جدول (2): ملذص النتائج لأبعاد حوض إمهوف المقترح للتجمع السكاني ألف وحدة سكنية

\begin{tabular}{|c|c|}
\hline \multicolumn{2}{|c|}{ الجزء العلوي من الحوض } \\
\hline${ }^{3}{ }^{3} 78.28$ & حجم الجزء العلوي \\
\hline 3.4 & العمق بدون المسافة الحرة \\
\hline 22.6 & معدل التحميل السطحي \\
\hline ا 3.8 م/ ساعة & السرعة الأفقية للمياه بالحوض \\
\hline 3.9 & العمق الكلي للحوض \\
\hline 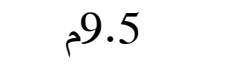 & طول الحوض \\
\hline 3.5 & عرض الحوض \\
\hline \multicolumn{2}{|c|}{ الجزء السفلي من الحوض } \\
\hline 5 & العرض الكلي للحوض \\
\hline 0.6 & عرض فتحات الغاز لكل جهة \\
\hline 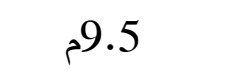 & طول الحوض \\
\hline${ }^{2} 1^{*} 0.75$ & مساحة القاعدة السفلي للهرم \\
\hline 2 & عمق الخزان من الاسفل للهرم بزاوية 45 \\
\hline $\begin{array}{l}3 \\
3\end{array}$ & حجم الهرم السفلي للحوض \\
\hline
\end{tabular}




\begin{tabular}{|r|r|}
\hline حمق الجزء السفلي من الحوض الجزء السفلي بدون عمق الهرم السفلي \\
\hline 30.65 & العمق الكلى للحوض 240 \\
\hline
\end{tabular}

جدول (3): جداول الكميات التقريبية لإنشاء حوض إمهوف وخنادق تصريف المياه الناتجة من التجمع

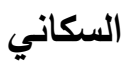

\begin{tabular}{|c|c|c|c|}
\hline 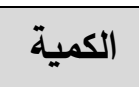 & 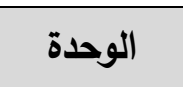 & 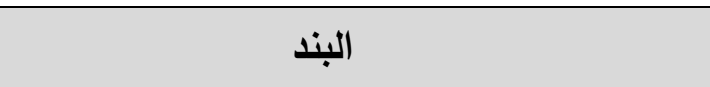 & م. \\
\hline 800 & $3_{3}^{3}$ & الأعمال الترابية لجميع مكونات منظومة المعالجة & 1 \\
\hline 100 & ${ }_{3}^{3}$ & أعمال الخرسانة لخزان إمهوف وخنادق التصريف & 2 \\
\hline 11000 & متر • طولي & أكمال توريد وتركيب مواسير البولي إثيلين عالي & 3 \\
\hline 50000 & مقطوعية & تكاليف متنوعة أخرى & 4 \\
\hline
\end{tabular}

| - الاستنتاجات

1. إن طرق المعالجة بالموقع واستخدام الطرق الطبيعية للمعالجة يعد أفضل من المعالجة المركزية من ناحية التكلفة الإجمالية والتشغيل والصيانة ويمكن استخدامها في حالة تعذر استخدام الطرق

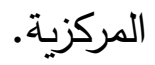

2. يجب إلا تثكل منظومات تجميع ومعالجة مياه الصرف الصحي التقليدية أو المركزية الخيار الوحيد المقبول لتجميع ومعالجة مياه الصرف الصحي حيث أن التركيز على هذا الخيار يعرقل أو يبطئ من انتثار الخدمة نتيجة التكاليف العالية المطلوبة، حيث يلزم التوجه الجدي نحو المعالجة المنفردة بالمكان للمنازل عبر نماذج حديثة وفعالة من أحواض التحليل واحواض إمهوف، والاستفادة من خبرة الدول المطبقة لأنظمة نقل مياه الصرف الصحي الحديثة الخاصة 
بالتجمعات القروية والتجمعات ذات المنازل المتباعدة أو ذات الطبيعة الصخرية التي توفر 50\% تقريباً من كلفة تتفيذ شبكة الصرف الصحي التقليدية.

3. خلال مرحلة تنفيذ المشروع يمكن تنفيذ حوض إمهوف، صندوق التوزيع، أما خنادق التصريف فيمكن تتفيذ جزء واحد منها كمرحلة أولى حيث يمكن تقسيم مساحة خنادق التصريف إلى أربعة أجزاء، وباقي الأجزاء يمكن تتفيذها في مراحل لاحقة. حسب الإمكانيات المادية بالمنطقة. 4. من خلال دراسة نظام الصرف الصحي للتجمع السكاني ألف وحدة سكنية ودراسة نظام الصرف الصحي المتبع بالمدينة يمكن تتفيذ إحدى الخيارات التالية لمعالجة مياه الصرف الصحي. تخصيص وتثغيل المحطة القائمة بالموقع المجاور للتجمع السكاني ألف وحدة سكنية لمعالجة مياه الصرف الصحي الناتج من التجمع السكاني ألف وحدة سكنية. وإعادة تصميم وإنشاء محطة أخرى لمعالجة التدفق الناتج من باقي أجزاء المدينة في مرحلة لاحقة ضمن مشروع متكامل للمدينة يشمل ربط المنازل، وخطوط تجميع وضخ مياه الصرف الصحي إلى

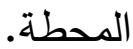
تصميم وإنشاء نظام معالجة يعمل بتقنية خزنات إمهوف لمعالجة التذفق الناتج من التجمع السكاني ألف وحدة سكنية الذي تم تقديره بـ (750 م ميوم) إلى حين يتم تشغيل المحطة القائمة للمدينة وربط التجمع السكاني بها.

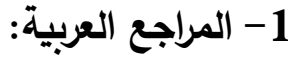

• التركماني، عبدالرزاق، 2009، الإدارة الهنسية لمياه الصرف في التجمعات السكانية الصغيرة.

• معهد علوم المياه بهولندا (UNESCO-IHE)، نوفمبر 2000، تحسين إدارة مياه الصرف الصحي

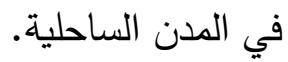

الفلاح، محمود 2005، مقترح استراتيجية عمل لمرافق معالجة مياه الصرف الصحي، المؤتمر الوطني الأول لتقنيات معالجة مياه الصرف الصحي والصناعي، بنغازي. 
• مكتب المعلومات 2014، الثركة العامة للمياه والصرف الصحي، طرابلس - ليبيا.

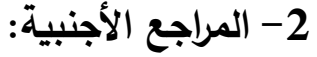

- Anne Mikelonis, may 15/2008, Water and Sanitation in Developing Countries Final Paper.

- Gijzen, H.J., 2003. A 3-step strategic approach to sewage management for sustainable water resources protection, Asian Water Quality.

- Metcalf \& Eddy Inc, 2003, Waste Water Engineering Treatment \& Disposal And Reuse. McGraw-Hill, $4^{\text {th }}$. Edition, New York.

- Imhof, B. and Muhlemann, J. 2005.Grey water Treatment on Household Level in Developing Countries.

- Lee boon, other, 2015, Reversing clogging in Imhoff tanks by catalyzed hydrogen peroxide treatment, Water Practice \& Technology, Vol 10 No 2.

- Yashashri Patil, 2018, Design of Sewage Treatment Plant Units for ST. John College Campus, International Journal of Scientific Research Engineering \& Technology, Volume 7, Issue 3, March 2018. 